\title{
Some Solutions for Electromagnetic Problems Involving Spheroidal, Spherical, and Cylindrical Bodies
}

\author{
James R. Wait
}

(September 29, 1959)

\begin{abstract}
Solutions are presented for the low-frequency electromagnetic response to an oscillating magnetic dipole by conducting bodies of simple shape. The quasi-stationary approximation is employed throughout, which is valid when the relevant dimensions of the problem are all small compared to the free-space wavelength. This amounts to matching solutions of the wave equation within the bodies to solutions of Laplace's equation outside. The results have application to geophysical prospecting.
\end{abstract}

\section{Introduction}

Electromagnetic methods of geophysical exploration utilize the fact that the conductivity of massive ore bodies is much greater than the surrounding barren rock. The general scheme is to set up a primary or exciting field by a current-carrying loop and then to detect the secondary field or response of the body by means of a receiving loop. The operating frequency should be sufficiently low that the attenuation by the surrounding barren rock is negligible. This usually requires frequencies in the audio range. The literature on the subject is extensive and here only certain representative papers are referenced [1-4]. ${ }^{1}$

Theoretical approaches to the subject are usually restricted to highly simplified situations. For example the exciting field is often assumed to be uniform or the body is taken to be perfectly conducting. While such solutions do indeed provide much useful information, there is a need to consider situations of a more general nature. For example, in the practical methods of electromagnetic prospecting the transmitting and receiving coils or loops may be located at distances from an ore body which are comparable to its maximum dimension and thus the uniform-exciting-field assumption is not valid. Furthermore, the frequency which is of the order of 500 cps is sufficiently low that the ore bodies seldom behave as if they were of perfect conductivity. For the above reasons, it seems worthwhile to set up solutions for certain idealized cases which do not suffer from such over-simplifying assumptions which are usually present. The geometrical forms considered are the prolate spheroid, the sphere, and the circular cylinder. Because of complexity the spheroid is taken to be perfectly conducting. The sphere and cylinder are assigned a conductivity and magnetic permeability which are finite. In each case the solution is presented for the case of an arbitrarily located magnetic dipole. The results are in a form which is suitable for computation.

In each case treated, special attention is paid to the equatorial plane which contains the source and observer. In the three instances, the source is at $C$ and the observer at $P$ as indicated in figure 1. For a (y-directed) magnetic dipole of strength $\overline{\mathrm{K}}$ at $C$, the primary field at $P$ is

$$
H_{y}^{p}=-\frac{\bar{K} d l}{2 \pi s^{3}}(\operatorname{amp} / \mathrm{m},)
$$

where $\overline{\mathrm{K}} d l$ is the magnetic moment. Note that $\overline{\mathrm{K}} d l=($ amp-turns $) \times($ coil area). In what follows the secondary fields are expressed in terms of $x$ (distance from coil axes to center of body), $y$ and $y^{\prime}$ (coordinates of source and observer), $s$ (distance between $C$ and $P$ ), $\sigma$ conductivity of body $(\mathrm{mhos} / \mathrm{m})$, and $\mu / \mu_{0}$ (magnetic permeability ratio).

1 Figures in brackets indicate the literature references at the end of this paper. 


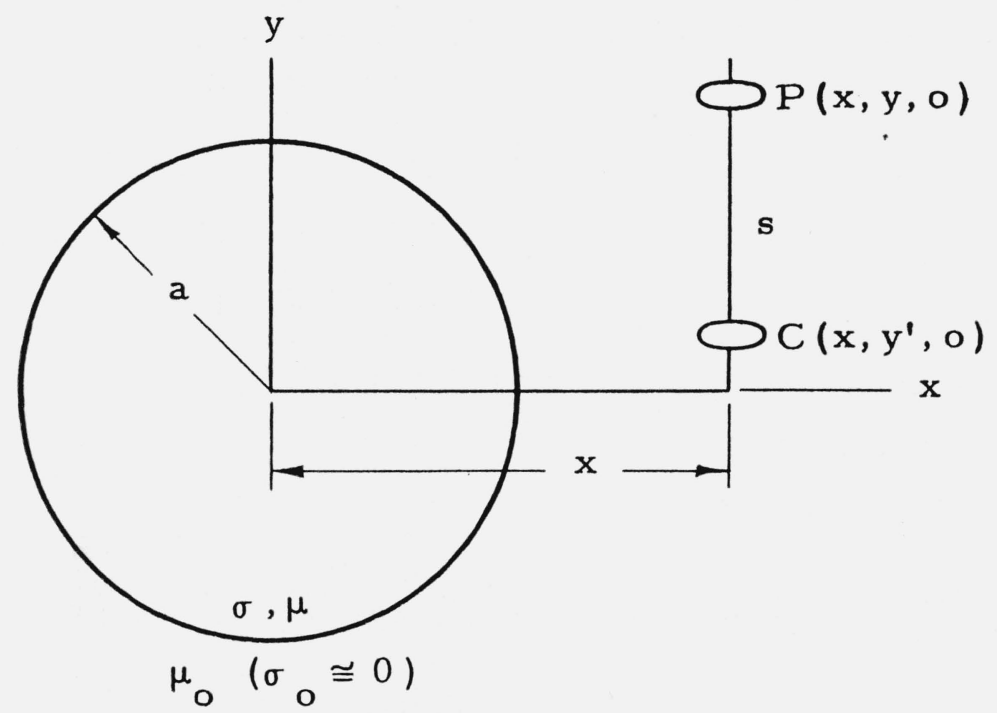

Figure 1.-Cross section of spheroid, cylinder, or sphere in equatorial plane $(z=0)$, showing transmitting and receiver coils.

\section{Low-Frequency Electromagnetic Response of a Highly-Conducting Spheroid}

Prolate spheroidal coordinates are introduced $(\rho, \eta, \delta)$. They are defined by

$$
\begin{array}{ll}
\frac{\rho^{2}}{c^{2}\left(\eta^{2}-1\right)}+\frac{z^{2}}{c^{2} \eta^{2}}=1 \quad & \text { (eq of spheroids), } \\
\frac{\rho^{2}}{-c^{2}\left(1-\delta^{2}\right)}+\frac{z^{2}}{c^{2} \delta^{2}}=1 \quad & \text { (eq of hyperboloids), }
\end{array}
$$

where $\rho$ and $z$ are the usual radial and axial coordinates in a cylindrical coordinate system. It thus follows that

$$
\rho=c\left[\left(1-\delta^{2}\right)\left(\eta^{2}-1\right)\right]^{\frac{1}{2}} \quad \text { and } \quad z=c \eta \delta .
$$

where $c$ is the semifocal distance. These prolate spheroidal coordinates are taken to be confocal with the spheroidal body whose surface is defined by $\eta=\eta_{0}$ (see fig. 2).

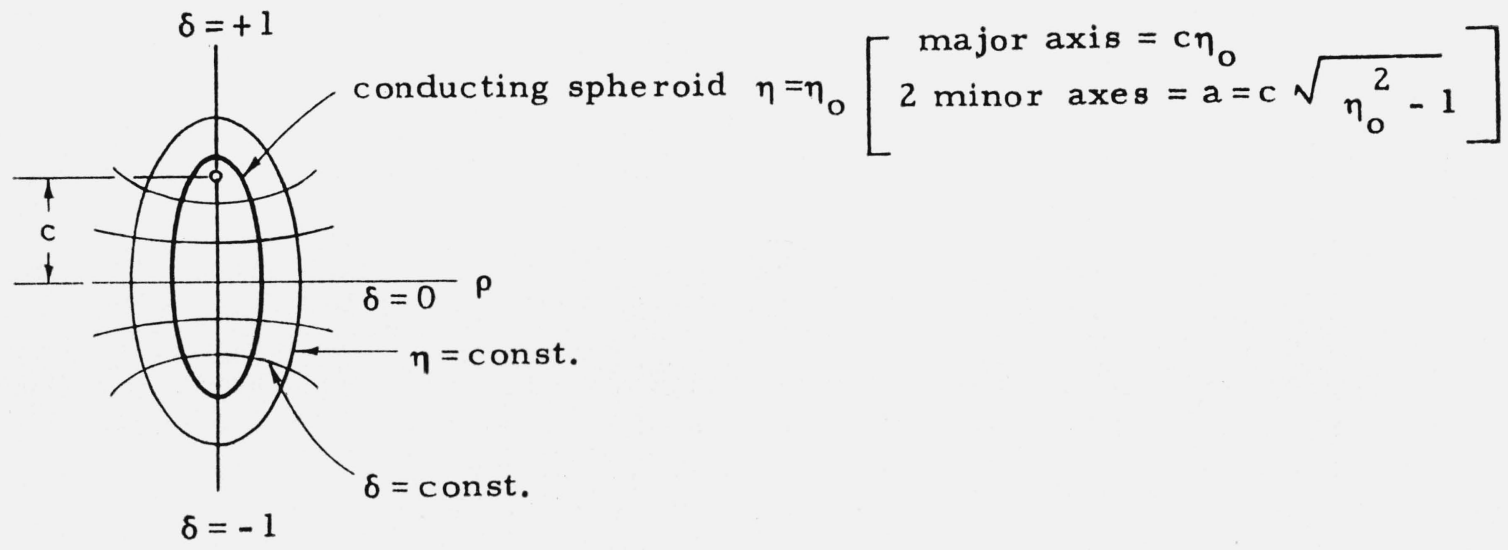

FIGURE 2.-The spheroid and spheroidal coordinate system. 
In the region exterior to the body (i.e., $\eta>\eta_{0}$ ) the fields are a solution of Laplace's equation if the frequency is sufficiently low (i.e., significant distances should be much less than the wavelength). Furthermore, in this case the fields can be derived from a magnetic potential $\Omega$. Thus,

$$
\vec{H}=-\operatorname{grad} \Omega
$$

where

$$
\nabla^{2} \Omega=0 \quad \text { for } \quad \eta>\eta_{0} .
$$

Now, solutions of Laplace's equation in spheroidal coordinates are well known and are of the form (for integral values of $m$ and $n$ )

$$
\left[A P_{n}^{m}(\delta)+B Q_{n}^{m}(\delta)\right]\left[A^{\prime} P_{n}^{m}(\eta)+B^{\prime} Q_{n}^{m}(\eta)\right] e^{ \pm \operatorname{mim} \phi},
$$

where $A, B, A^{\prime}$, and $B^{\prime}$ are constants and, following Smythe [5], $P_{n}^{m}(\mu)$ and $Q_{n}^{m}(\mu)$ are associated Legendre functions of argument $\mu$. They are defined by

or

$$
\left.\begin{array}{l}
P_{n}^{m}(\mu)=\left(\mu^{2}-1\right)^{m / 2} \frac{d^{m} P_{n}(\mu)}{d \mu^{m}} \\
Q_{n}^{m}(\mu)=\left(\mu^{2}-1\right)^{m / 2} \frac{d^{m} Q_{n}(\mu)}{d \mu^{m}}
\end{array}\right] \text { for } \mu>1
$$

$$
\left.\begin{array}{l}
P_{n}^{m}(\mu)=\left(1-\mu^{2}\right)^{m / 2} \frac{d^{m} P_{n}(\mu)}{d \mu^{m}} \\
Q_{n}^{m}(\mu)=\left(1-\mu^{2}\right)^{m / 2} \frac{d^{m} Q_{n}(\mu)}{d \mu^{m}}
\end{array}\right] \text { for }-1<\mu<1
$$

in terms of the (ordinary) Legendre functions $P_{n}(\mu)$ and $Q_{n}(\mu)$. It should be noted Hobson introduces a factor $(-1)^{m}$ on the right-hand side of the latter two equations and his definitions are thus slightly different.

At the point $C$ it is assumed that there is a magnetic point charge of strength, $\bar{K}$ (see fig. 3). The magnetic potential at $P$ can now be written

$$
\Omega=\frac{\bar{K}}{4 \pi R}+\Omega^{s}
$$

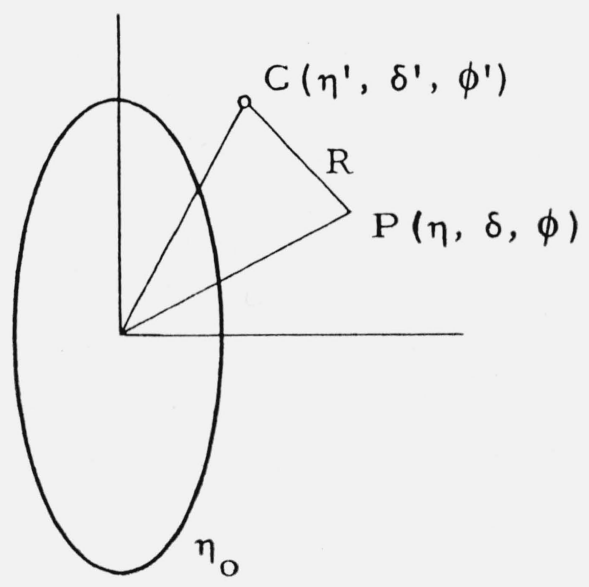

FIgure 3.-Conducting spheroid and source $C$ and observer $P$. 
where $R$ is the linear distance between $C$ at $\left(\eta^{\prime}, \delta^{\prime}, \phi^{\prime}\right)$ and $P$ at $(\eta, \delta, \phi)$ and $\Omega^{s}$ is the secondary influence of the body. To express $\Omega$ in prolate-spheroidal coordinates, we make use of the inverse-distance formula

$$
\frac{1}{R}=\frac{1}{c} \sum_{n=0}^{\infty} \sum_{m=0}^{n}\left(2-\delta_{m}\right)(-1)^{m}\left[\frac{(n-m) !}{(n+m) !}\right]^{2}(2 n+1) P_{n}^{m}(\delta) Q_{n}^{m}\left(\eta^{\prime}\right) P_{n}^{m}(\eta) P_{n}^{m}\left(\delta^{\prime}\right) \cos m\left(\phi-\phi^{\prime}\right),
$$

which is valid for $\eta<\eta^{\prime}$. [ $\left.\delta_{0}=1, \delta_{m}=0(m \neq 0)\right]$.

The potential $\Omega$ is a solution of Laplace's equation and since $1 / R$ is also, it follows that $\nabla^{2} \Omega^{s}=0$ for $\eta>\eta_{0}$. Thus $\Omega^{s}$ must contain terms of the type

$$
Q_{n}^{m}(\eta) P_{n}^{m}(\delta) \cos m\left(\phi-\phi^{\prime}\right)
$$

only since $P_{n}^{m}(\eta)$ is infinite at $\eta \rightarrow \infty$ and $Q_{n}^{m}(\delta)$ is infinite at $\delta= \pm 1$. The potential exterior to the spheroid is thus written, for $\eta<\eta^{\prime}$,

$$
\Omega=\frac{\bar{K}}{4 \pi} \sum_{n=0}^{\infty} \sum_{m=0}^{n}\left[M_{m n} P_{n}^{m}(\eta)+B_{m n} Q_{n}^{m}(\eta)\right] P_{n}^{m}(\delta) \cos m\left(\phi-\phi^{\prime}\right),
$$

where

$$
M_{m n}=\frac{1}{c}\left(2-\delta_{m}\right)(-1)^{m}(2 n+1)\left[\frac{(n-m) !}{(n+m) !}\right]^{2} Q_{n}^{m}\left(\eta^{\prime}\right) P_{n}^{m}\left(\delta^{\prime}\right) .
$$

The unknown coefficient is now found from the boundary condition, that on the surface of the spheroid the normal component of the total magnetic field must vanish. Thus,

This is satisfied if

$$
\frac{\partial \Omega}{\partial \eta}=0 \quad \text { at } \quad \eta=\eta_{0}
$$

$$
B_{m n}=-M_{m n} \frac{P_{n}^{m^{\prime}}\left(\eta_{0}\right)}{Q_{n}^{m^{\prime}}\left(\eta_{0}\right)}
$$

where the prime indicates the derivative with respect to the argument of the Legendre function. The solution is thus given by

$$
\Omega=\frac{\bar{K}}{4 \pi}\left[\frac{1}{R}-\sum_{n=0}^{\infty} \sum_{m=0}^{n} M_{m n} \frac{P_{n}^{m^{\prime}}\left(\eta_{0}\right)}{Q_{\mathrm{n}}^{m^{\prime}}\left(\eta_{0}\right)} Q_{n}^{m}(\eta) P_{n}^{m}(\delta) \cos m\left(\phi-\phi^{\prime}\right)\right]
$$

valid for $\eta>\eta_{0}$. To simplify things a bit we will consider our source to be a $y$-directed magnetic dipole $T$ located in the equatorial plane of the spheroid $(z=0)$ (see fig. 4).

The potential at $R(x, y, z)$ is thus given by

$$
\begin{aligned}
\Phi & =-d l \frac{\partial}{\partial y^{\prime}} \Omega \\
& =+\frac{\bar{K} d l}{4 \pi}\left\{-\frac{\partial}{\partial y^{\prime}} \frac{1}{R}+\sum_{n=0}^{\infty} \sum_{m=0}^{n} \frac{P_{n}^{m^{\prime}}\left(\eta_{0}\right)}{Q_{n}^{m \prime}\left(\eta_{0}\right)} Q_{n}^{m}(\eta)\right. \\
& \left.\times P_{n}^{m}(\delta)\left[\frac{\partial M_{m n}}{\partial y^{\prime}} \cos m\left(\phi-\phi^{\prime}\right)+\frac{\partial}{\partial y^{\prime}} \cos m\left(\phi-\phi^{\prime}\right) \cdot M_{m n}\right]\right\} .
\end{aligned}
$$

The magnetic field at $R$ is obtained from

$$
\vec{H}=-\operatorname{grad} \Phi .
$$

In the case of the $y$-component, for example, we have

$$
H_{y}=-\frac{\partial \Phi}{\partial y}=d l \frac{\partial^{2} \Omega}{\partial y^{\prime} \partial y} .
$$




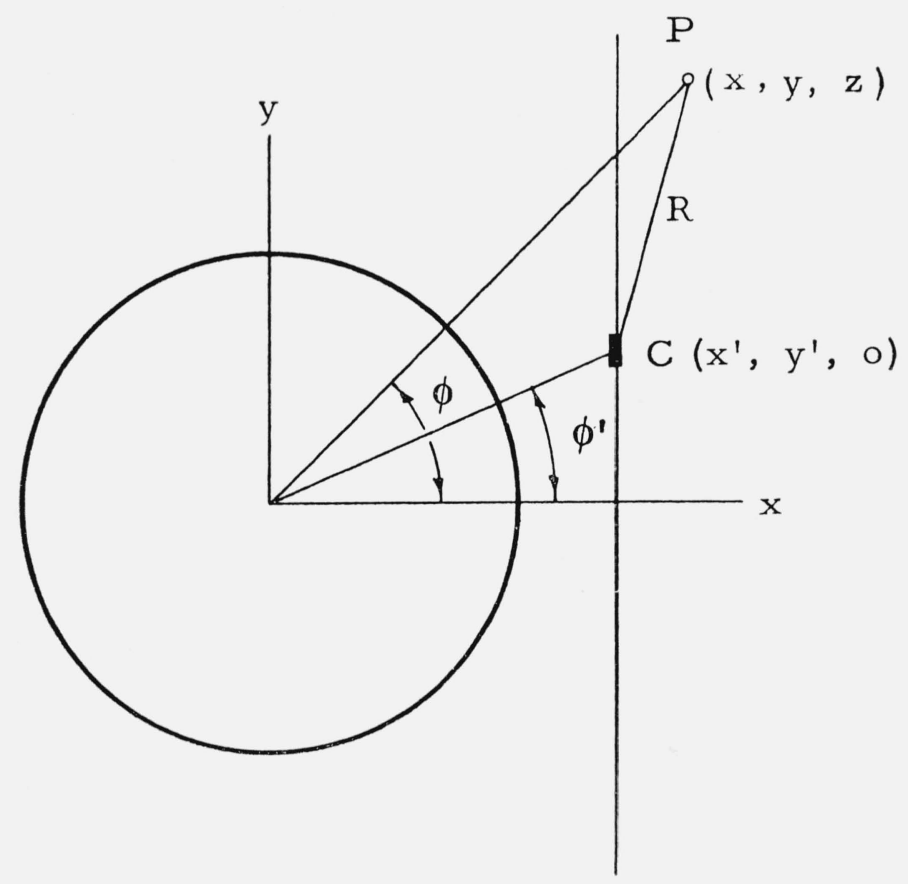

FIGURE 4.-The conducting spheroid with locations of source $C$ and observer $P$.

But

$$
\frac{\partial \Omega}{\partial y}=\frac{\partial \Omega}{\partial \eta} \cdot \frac{\partial \eta}{\partial y}+\frac{\partial \Omega}{\partial \phi} \cdot \frac{\partial \phi}{\partial y}
$$

and therefore

$$
\frac{\partial^{2} \Omega}{\partial y \partial y^{\prime}}=\frac{\partial^{2} \Omega}{\partial \eta^{\prime} \partial \eta}\left(\frac{\partial \eta}{\partial y}\right)\left(\frac{\partial \eta^{\prime}}{\partial y^{\prime}}\right)+\frac{\partial^{2} \Omega}{\partial \phi^{\prime} \partial \phi}\left(\frac{\partial \phi}{\partial y}\right)\left(\frac{\partial \phi^{\prime}}{\partial y^{\prime}}\right)+\frac{\partial^{2} \Omega}{\partial \phi^{\prime} \partial \eta}\left(\frac{\partial \eta}{\partial y}\right)\left(\frac{\partial \phi^{\prime}}{\partial y^{\prime}}\right)+\frac{\partial^{2} \Omega}{\partial \eta^{\prime} \partial \phi}\left(\frac{\partial \phi}{\partial y}\right)\left(\frac{\partial \eta^{\prime}}{\partial y^{\prime}}\right) .
$$

Since

$$
y=c \sqrt{\left(1-\delta^{2}\right)\left(\eta^{2}-1\right)} \sin \phi
$$

and

$$
x=c \sqrt{\left(1-\delta^{2}\right)\left(\eta^{2}-1\right)} \cos \phi,
$$

it readily follows that for $\delta=0$ (or $z=0$ ),

$$
\frac{\partial \eta}{\partial y}=\frac{\sin \phi}{c} \frac{\sqrt{\eta^{2}-1}}{\eta} \quad \text { and } \quad \frac{\partial \phi}{\partial y}=\frac{\cos \phi}{c} \frac{1}{\sqrt{\eta^{2}-1}}
$$

and similarly for the primed quantities. Thus,

$$
H_{y}=d l \frac{\partial^{2} \Omega}{\partial y^{\prime} \partial y}
$$

for a $y$-directed dipole in the plane $z=0$,

$$
\Omega=\frac{\bar{K}}{4 \pi R}+\Omega^{s},
$$


where

$$
\Omega^{s}=-\frac{\bar{K}}{4 \pi c} \sum_{n=0}^{\infty} \sum_{m=0}^{n} \epsilon_{m}(-1)^{m}(2 n+1)\left[\frac{(r-m !)}{(n+m) !}\right]^{2} \frac{P_{n}^{m^{\prime}}\left(\eta_{0}\right)}{Q_{n}^{m^{\prime}}\left(\eta_{0}\right)}\left[P_{n}^{m}(0)\right]^{2} \cos m\left(\phi-\phi^{\prime}\right) Q_{n}^{m}\left(\eta^{\prime}\right) Q_{n}^{m}(\eta)
$$

where

$$
\boldsymbol{\epsilon}_{m}=2-\delta_{m}=1 \quad \text { for } \quad m=0
$$

and

$$
\delta=\delta^{\prime}=0
$$

Now

$$
\begin{aligned}
\frac{\partial^{2}}{\partial y \partial y^{\prime}} Q(\eta) Q\left(\eta^{\prime}\right) & \cos m\left(\phi-\phi^{\prime}\right)=V_{n}^{m}\left(\eta, \eta^{\prime}, \phi-\phi^{\prime}\right) \\
& =Q^{\prime}(\eta) Q^{\prime}\left(\eta^{\prime}\right) \cos m\left(\phi-\phi^{\prime}\right)\left(\frac{\sin \phi}{c} \frac{\sqrt{\eta^{2}-1}}{\eta}\right)\left(\frac{\sin \phi^{\prime}}{c} \frac{\sqrt{\left(\eta^{\prime}\right)^{2}-1}}{\eta^{\prime}}\right) \\
& \left.+Q(\eta) Q\left(\eta^{\prime}\right) m^{2} \cos m^{\prime} \phi-\phi^{\prime}\right)\left(\frac{\cos \phi}{c} \frac{1}{\sqrt{\eta^{2}-1}}\right)\left(\frac{\cos \phi^{\prime}}{c} \frac{1}{\sqrt{\left(\eta^{\prime}\right)^{2}-1}}\right) \\
& -Q(\eta) Q^{\prime}\left(\eta^{\prime}\right) m \sin m\left(\phi-\phi^{\prime}\right)\left(\frac{\cos \phi}{c} \frac{1}{\sqrt{\eta^{2}-1}}\right)\left(\frac{\sin \phi^{\prime}}{c} \frac{\sqrt{\left(\eta^{\prime}\right)^{2}-1}}{\eta^{\prime}}\right) \\
& +Q^{\prime}(\eta) Q\left(\eta^{\prime}\right) m \sin m\left(\phi-\phi^{\prime}\right)\left(\frac{\sin \phi}{c} \frac{1}{\sqrt{\eta^{2}-1}}\right)\left(\frac{\cos \phi^{\prime}}{c} \frac{1}{\sqrt{\left(\eta^{\prime}\right)^{2}-1}}\right)
\end{aligned}
$$

where $Q=Q_{n}^{m}$.

Finally,

where

$$
H_{y}=H_{y}^{p}+H_{y}^{s}
$$

$$
\begin{aligned}
H_{y}^{p} & =\frac{\bar{K} d l}{4 \pi} \frac{\partial^{2}}{\partial y \partial y^{\prime}} \frac{1}{R}=-\frac{\bar{K} d l}{4 \pi}\left[\frac{3\left(y-y^{\prime}\right)^{2}}{R^{5}}-\frac{1}{R^{3}}\right] \\
& =-\frac{\bar{K} d l}{2 \pi s^{3}} \quad \text { for } \quad x^{\prime}=x .
\end{aligned}
$$

Noting that

and

$$
R=\left|y^{\prime}-y\right|=s=y-y^{\prime}
$$

$$
H_{y}^{s}=-\frac{\bar{K}}{4 \pi c} \sum_{n=0}^{\infty} \sum_{m=0}^{n} \epsilon_{m}(-1)^{m}(2 n+1)\left[\frac{(n-m) !}{(n+m) !}\right]^{2} \frac{P_{n}^{m^{\prime}}\left(\eta_{0}\right)}{Q_{n}^{m^{\prime}}\left(\eta_{0}\right)}\left[P_{n}^{m}(0)\right]^{2} V_{n}^{m}
$$

where $V_{n}^{m}$ is as given above.

For the special case (i.e., $z=z^{\prime}=0,\left|y^{\prime}-y\right|=s$, and $x=x^{\prime}$ ) as shown in figure 5, we have

$$
a^{2}=c^{2}\left(\eta_{0}^{2}-1\right), \quad a=c \sqrt{\eta_{0}^{2}-1},
$$




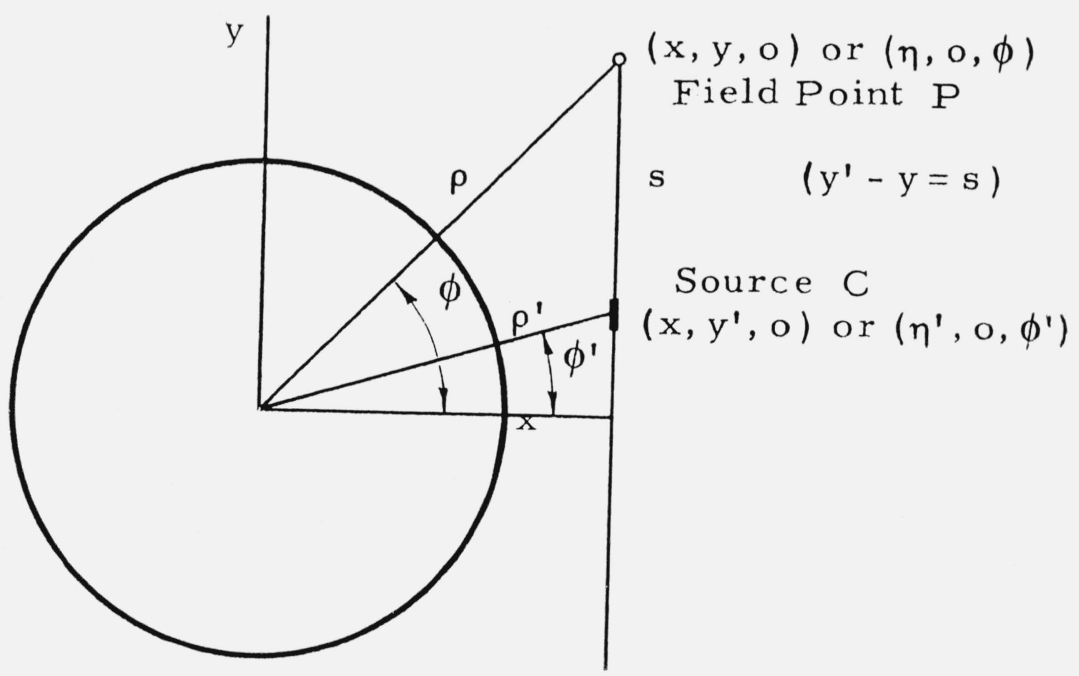

FIGURE 5.- Special configuration of (dipole) source $C$ and observer $P$ when they are located in equatorial plane of spheroid.

where $a$ is the semiminor axis, $c$ is the semifocal distance,

$$
\rho^{2}=c^{2}\left(\eta^{2}-1\right), \quad \rho=c \sqrt{\eta^{2}-1}
$$

and

$$
\left(\rho^{\prime}\right)^{2}=c^{2}\left[\left(\eta^{\prime}\right)^{2}-1\right], \quad \rho^{\prime}=c \sqrt{\left(\eta^{\prime}\right)^{2}-1}
$$

Also it should be noted that

$$
\begin{gathered}
s=\left[\rho^{2}+\left(\rho^{\prime}\right)^{2}-2 \rho \rho^{\prime} \cos \left(\phi-\phi^{\prime}\right)\right]^{\frac{1}{2}}, \\
\tan \phi^{\prime}=\frac{y^{\prime}}{x} \quad \text { and } \quad \tan \phi=\frac{y}{x} .
\end{gathered}
$$

If $b$ is the semimajor axis,

$$
b=c \eta_{0} .
$$

\section{Low-Frequency Electromagnetic Response of a Finitely-Conducting Sphere With Arbitrary Permeability}

Spherical coordinates $(r, \theta, \phi)$ are introduced as indicated in figure 6 . The surface of a spherical body (of conductivity $\sigma$ and permeability $\mu$ ) is defined by $r=a$. Exterior to the sphere the fields can be derived from a magnetic potential $\Omega$. Thus,

where

$$
\left.\begin{array}{l}
H=-\operatorname{grad} \Omega \\
\nabla^{2} \Omega=0
\end{array}\right\} \text { for } r>a .
$$

Inside the sphere the fields are a solution of the wave equation and are best derived in terms of scalar stream functions (following Schelkunoff) [6]. In the present problem we need only 




FIGURE 6.-The spherical body and source $C$ and observer $P$ with the spherical coordinate system.

consider the TE modes which are associated with the induced magnetic dipoles. Thus,

$$
\left.\begin{array}{rl}
H_{r} & =\frac{1}{i \mu \omega}\left(\frac{\partial^{2}}{\partial r^{2}}-\gamma^{2}\right) \psi \\
& =\frac{1}{i \mu \omega r^{2}}\left[\frac{1}{\sin \theta} \frac{\partial}{\partial \theta}\left(\sin \theta \frac{\partial}{\partial \theta}\right)+\frac{1}{\sin ^{2} \theta} \frac{\partial^{2}}{\partial \phi^{2}}\right] \psi \\
H_{\theta} & =\frac{1}{i \mu \omega} \frac{\partial^{2} \psi}{\partial r \partial \theta} \\
H_{\phi} & =\frac{1}{i \mu \omega r \sin \theta} \frac{\partial^{2} \psi}{\partial r \partial \phi}
\end{array}\right\}
$$

where $\psi$ is the stream function and

whereas

$$
\gamma=[i \mu \omega(\sigma+i \omega \epsilon)]^{\frac{1}{2}} \cong(i \sigma \mu \omega)^{\frac{1}{2}}
$$

$$
\begin{aligned}
H_{r} & =\frac{1}{i \mu_{0} \omega} \frac{\partial^{2}}{\partial r^{2}} \psi_{0}=-\frac{\partial \Omega}{\partial r} \\
& =\frac{1}{i \mu_{0} \omega r^{2}}\left[\frac{1}{\sin \theta} \frac{\partial}{\partial \theta}\left(\sin \theta \frac{\partial}{\partial \theta}\right)+\frac{1}{\sin ^{2} \theta} \frac{\partial^{2}}{\partial \phi^{2}}\right] \psi_{0}, \\
H_{\theta} & =\frac{1}{i \mu_{0} \omega r} \frac{\partial^{2} \psi_{0}}{\partial r \partial \theta}=-\frac{\partial \Omega}{r \partial \theta}, \\
H_{\phi} & =\frac{1}{i \mu_{0} \omega r \sin \theta} \frac{\partial^{2} \psi_{0}}{\partial r \partial \phi}=-\frac{\partial \Omega}{r \sin \theta \partial \phi},
\end{aligned}
$$

where $\psi_{0}$ is the stream function pertinent to the exterior region and is related to the magnetic potential by

$$
\Omega=-\frac{1}{i \mu_{0} \omega} \frac{\partial \psi_{0}}{\partial r}
$$


The stream functions satisfy

$$
\left[r^{2} \frac{\partial^{2}}{\partial r^{2}}+\frac{1}{\sin \theta} \frac{\partial}{\partial \theta}\left(\sin \theta \frac{\partial}{\partial \theta}\right)+\frac{1}{\sin ^{2} \theta} \frac{\partial^{2}}{\partial \phi^{2}}\right] \begin{aligned}
& \psi=\gamma^{2} r^{2} \psi \\
& \psi_{0}=0
\end{aligned} \text { for } \quad \text { for } r \quad<a
$$

Thus they are of the form

and

$$
\begin{aligned}
& \hat{I}_{n}(\gamma r) \\
& \hat{K}_{n}(\gamma r)
\end{aligned} P_{n}^{m}(\cos \theta) \quad \text { for } \quad r<a
$$

$$
r^{+(n+1)} P_{n}^{m}(\cos \theta) \quad \text { for } \quad r>a
$$

In the above $\hat{I}_{n}$ and $\hat{K}_{n}$ are the spherical modified Bessel functions defined by

$$
\hat{I}_{n}(z)=\frac{1}{2}\left[e^{z} \sum_{m=0}^{n} \frac{(-1)^{m}(n+m) !}{m !(n-m) !(2 z)^{m}}+(-1)^{n+1} e^{-z} \sum_{m=0}^{n} \frac{(n+m) !}{m !(n-m) !(2 z)^{m}}\right]
$$

and

$$
\hat{K}_{n}(z)=e^{-z} \sum_{m=0}^{n} \frac{(n+m) !}{m !(n-m) !(2 z)^{m}}
$$

following Schelkunoff [6].

Now for $r>a$,

$$
\Omega=\Omega^{p}+\Omega^{3}
$$

where the primary source is denoted $\Omega^{p}$ and the influence of the sphere by $\Omega^{s}$. Assuming a magnetic charge (strength $\overline{\mathrm{K}}$ ) located at point $C$ with coordinates $\left(r^{\prime}, \theta^{\prime}, \phi^{\prime}\right)$, the potential $\Omega^{p}$ at point $P$ with coordinates $(r, \theta, \phi)$ is given by

$$
\begin{aligned}
\Omega^{p} & =\frac{\bar{K}}{4 \pi R} \\
& =\frac{\bar{K}}{4 \pi} \sum_{n=0}^{\infty} \sum_{m=0}^{n} \epsilon_{m} \frac{(n-m) !}{(n+m) !} \frac{r^{n}}{\left(r^{\prime}\right)^{n+1}} P_{n}^{m}(\cos \theta) P_{n}^{m}\left(\cos \theta^{\prime}\right) \times \cos m\left(\phi-\phi^{\prime}\right)
\end{aligned}
$$

for $r<r^{\prime}$, using a well-known addition theorem for the inverse distance in spherical harmonics [5]. This suggests writing

$$
\Omega^{s}=\frac{\bar{K}}{4 \pi} \sum_{n=0}^{\infty} \sum_{m=0}^{n} \epsilon_{m} \frac{(n-m) !}{(n+m) !}\left(\frac{n}{n+1}\right) S_{n} \frac{a^{2 n+1}}{\left(r^{\prime}\right)^{n+1} r^{n+1}} \times P_{n}^{m}(\cos \theta) P_{n}^{m}\left(\cos \theta^{\prime}\right) \cos m\left(\phi-\phi^{\prime}\right),
$$

where $S_{n}$ is an undetermined coefficient. The appropriate form for $\psi_{0}$ is thus

$$
\psi_{0}=-\frac{\bar{K} i \mu_{0} \omega}{4 \pi} \sum_{n=0}^{\infty} \sum_{m=0}^{n} \epsilon_{m} \frac{(n-m) !}{(n+m) !}\left\{\left(\frac{r}{r^{\prime}}\right)^{n+1}-\frac{a^{2 n+1}}{\left(r^{\prime}\right)^{n+1} r^{n}} S_{n}\right\} \frac{P_{n}^{m}(\cos \theta) P_{n}^{m}\left(\cos \theta^{\prime}\right)}{(n+1)} \times \cos m\left(\phi-\phi^{\prime}\right) .
$$

For the interior the solution must be of the form

$$
\psi=-\frac{\bar{K} i \mu_{n} \omega}{4 \pi} \sum_{n=0}^{\infty} \sum_{m=0}^{n} \epsilon_{m} \frac{(n-m) ! \hat{I}_{n}(\gamma r)}{(n+m) ! \hat{I}_{n}(\gamma a)} \frac{P_{n}^{m}(\cos \phi) P_{n}^{m}\left(\cos \phi^{\prime}\right)}{(n+1)} B_{m}\left(\frac{a}{r^{\prime}}\right)^{n+1},
$$

where the $\hat{K}_{n}(\gamma r)$ solution has been rejected since it becomes infinite at $r=0$ and where $B_{n}$ is an undetermined coefficient. 
The boundary conditions require that the normal flux $\left(\mu H_{r}\right)$ inside the sphere is continuous with the exterior normal flux $\left(\mu_{0} H_{r}\right)$ at $r=a$. Also the tangential fields $H_{\theta}$ and $H_{\phi}$ must be continuous at $r=a$. These conditions are satisfied if

$$
\left.\left.\mu \frac{\partial \psi}{\partial r}\right]_{r=a}=\mu_{0} \frac{\partial \psi_{0}}{\partial r}\right]_{r=a}
$$

and

$$
\left.\psi] \underset{r=a}{=} \psi_{0}\right]
$$

This leads to

$$
S_{n}=\frac{A_{n}(\alpha)-(n+1)\left(\mu / \mu_{0}\right)}{A_{n}(\alpha)+n\left(\mu / \mu_{0}\right)}
$$

where

$$
A_{n}(\alpha)=\frac{\alpha \frac{d \hat{I}_{n}(\alpha)}{d \alpha}}{\hat{I}_{n}(\alpha)}=\alpha \frac{d}{d \alpha}\left[\log _{e} \hat{I}_{n}(\alpha)\right]
$$

with $\alpha=\gamma a$. Also,

$$
B_{n}=\frac{\left(\mu / \mu_{0}\right)}{n\left(\mu / \mu_{0}\right)+A_{n}(\alpha)} .
$$

The above expressions can now be used to obtain an expression for the magnetic potential due to a dipole for $r>a$ in terms of the potential $\Omega$ due to a point charge. For example in the case of a $y$ directed dipole at $\left(r^{\prime}, \phi^{\prime}, z^{\prime}\right)$ we have

$$
\Phi_{0}=-d l \frac{\partial}{\partial y^{\prime}} \Omega
$$

The corresponding magnetic fields are thus given by

and the $y$-component, for example, is

$$
\vec{H}=-\operatorname{grad} \Phi_{0}
$$

$$
H_{y}=d l \frac{\partial \Omega}{\partial y \partial y^{\prime}} .
$$

Again we shall consider the equatorial plane (see fig. 7) which is $z=z^{\prime}=0$ or

$$
\begin{array}{rlrl}
\theta=\theta^{\prime}=\pi / 2 . & \text { Here } x & =r \cos \phi & x^{\prime}=r^{\prime} \cos \phi^{\prime} \\
y & =r \sin \phi & y^{\prime}=r^{\prime} \sin \phi^{\prime},
\end{array}
$$

and thus,

$$
\frac{\partial}{\partial y}=\sin \phi \frac{\partial}{\partial r}+\cos \phi \frac{\partial}{\rho \partial \phi}
$$

and

$$
\frac{\partial}{\partial y^{\prime}}=\sin \phi^{\prime} \frac{\partial}{\partial r^{\prime}}+\cos \phi^{\prime} \frac{\partial}{\rho^{\prime} \partial \phi^{\prime}}
$$

The field is thus obtained conveniently from

$$
H_{y}=d l\left[\sin \phi^{\prime} \sin \phi \frac{\partial^{2}}{\partial r^{\prime} \partial r}+\sin \phi^{\prime} \cos \phi \frac{\partial^{2}}{\rho \partial r^{\prime} \partial \phi}+\cos \phi^{\prime} \sin \phi \frac{\partial^{2}}{\rho^{\prime} \partial \phi^{\prime} \partial r}+\cos \phi^{\prime} \cos \phi \frac{\partial^{2}}{\rho^{\prime} \rho \partial \phi \partial \phi^{\prime}}\right] \Omega .
$$


It should be noted that

Writing

$$
\begin{array}{lll}
\rho^{\prime}=r^{\prime} & \text { for } & \theta^{\prime}=\pi / 2 \\
\rho=r & \text { for } & \theta=\pi / 2 .
\end{array}
$$

it is seen that

$$
H_{y}=H_{y}^{p}+H_{y}^{s},
$$

$$
H_{y}^{p}=\bar{K} \frac{d l}{4 \pi} \frac{\partial^{2}}{\partial y \partial y^{\prime}} \frac{1}{R} \quad \text { where } \quad R=\sqrt{\left(x-x^{\prime}\right)^{2}+\left(y-y^{\prime}\right)^{2}} \quad \text { for } \quad z=z^{\prime}=0,
$$

and thus,

$$
\begin{aligned}
H_{y}^{p} & =\frac{\bar{K} d l}{4 \pi} \frac{\partial}{\partial y^{\prime}}\left(\frac{y-y^{\prime}}{R^{3}}\right)=-d l\left[-\frac{1}{R^{3}}+\frac{3\left(y-y^{\prime}\right)^{2}}{R^{5}}\right] \frac{\bar{K}}{4 \pi} \\
& =-\frac{\bar{K} d l}{4 \pi s^{3}} \quad \text { where } \quad s=\left|y-y^{\prime}\right| .
\end{aligned}
$$

The secondary field is derived from

$$
\Omega_{0}^{s}=\frac{\bar{K}}{4 \pi} \sum_{n=0}^{\infty} \sum_{m=0}^{n} \epsilon_{m} \frac{(n-m) !}{(n+m) !} \frac{a^{2 n+1} n}{\left(r^{\prime}\right)^{n+1} r^{n+1}} S_{n} \frac{P_{n}^{m}(\cos \theta) P_{n}^{m}\left(\cos \theta^{\prime}\right)}{n+1} \cos m\left(\phi-\phi^{\prime}\right) .
$$

Finally (for $r \geqq a)$

where

$$
H_{y}^{s}=\frac{\bar{K}}{4 \pi} \sum_{n=0}^{\infty} \sum_{m=0}^{n} \epsilon_{m} \frac{(n-m) !}{(n+m) !} \frac{n}{n+1} S_{n}\left[P_{n}^{m}(0)\right]^{2} U_{n m} \frac{a^{2 n+1}}{\left(r^{\prime}\right)^{n+1} r^{n+1}}
$$

where

$$
\begin{aligned}
& U_{n m}=\frac{1}{r r^{\prime}}\left[(n+1)^{2} \sin \phi^{\prime} \sin \phi \cos m\left(\phi-\phi^{\prime}\right)\right. \\
&+m(n+1) \sin \phi^{\prime} \cos \phi \sin m\left(\phi-\phi^{\prime}\right) \\
& \quad-m(n+1) \cos \phi^{\prime} \sin \phi \sin m\left(\phi-\phi^{\prime}\right) \\
&\left.+m^{2} \cos \phi^{\prime} \cos \phi \cos m\left(\phi-\phi^{\prime}\right)\right],
\end{aligned}
$$

$$
\begin{aligned}
\tan \phi & =\frac{y}{x}, \\
\tan \phi^{\prime} & =\frac{y^{\prime}}{x} .
\end{aligned}
$$

The quantity $S_{n}$ plays rather an essential role in the final result. When the conductivity is great or at high frequency, the argument $\gamma a$ of the Bessel functions may be large. In fact if $|\gamma a|>>1, S_{n}$ approaches unity for all $n$. The behavior of $S_{n}$, as a function of $\gamma a$ and $\mu / \mu_{0}$, is a convenient way to illustrate the frequency dependence of the secondary fields. Numerical values of the real and imaginary parts of $S_{n}$ are given in some detail for $n=1$ in previous papers [2, 8] and less adequately [9] for $n=2,3$, and 4. Fortunately, if the source $C$ or the observer $P$ (for any respective orientations) are a distance from the center of the sphere which is large compared to the radius, it is seen that only the term corresponding to $S_{1}$ is important. The response of the sphere is thus proportional to the quantity

$$
S_{1}=\frac{\gamma a \hat{I}_{1}^{\prime}(\gamma a)-2 K \hat{I}_{1}(\gamma a)}{\gamma a \hat{I}_{1}^{\prime}(\gamma a)+K \hat{I}_{1}(\gamma a)} \quad \text { where } \quad K=\mu / \mu_{0} .
$$

The real and imaginary parts of $S_{1}$ are plotted in figure 8 as a function of the "relative radius" $X$ defined by

$$
\gamma a=\sqrt{i} X \quad \text { or } \quad X=(\sigma \mu \omega)^{\frac{1}{2}} a .
$$


For small values of $X$ corresponding to the lower frequencies, $S_{1}$ is negative and, in fact, it approaches the value $-2(K-1) /(K+2)$. This could have been derived directly from magnetostatics. At the higher frequencies $S_{1}$ approaches unity which corresponds to no penetration of magnetic flux into the body.

\section{Low-Frequency Response of a Conducting Cylinder With Arbitrary Permeability}

We now wish to calculate the electromagnetic response of an infinitely long conducting cylinder with any permeability. (See fig. 9.) Exterior to the cylinder $\rho>a$, the fields are a solution of Laplace's equation and are derivable from a potential. Inside the cylinder the fields are a solution of the wave equation and are derivable from a magnetic Hertz vector $\vec{\Pi}^{*}$ and an electric Hertz vector $\vec{\Pi}$ with only z-components. Thus (for $r<a$ )

$$
\vec{H}=\left(-\gamma^{2}+\operatorname{grad} \operatorname{div}\right) \vec{\Pi}^{*}+\frac{\gamma^{2}}{i \mu \omega} \operatorname{curl} \vec{\Pi},
$$

where

$$
\vec{\Pi}^{*}=\left(0,0, \Pi_{z}^{*}\right)
$$

and

$$
\vec{\Pi}=\left(0,0, \Pi_{z}\right) .
$$

Solutions of the wave equation

$$
\left(\nabla^{2}-\gamma^{2}\right)_{\Pi_{z}}^{\Pi_{z}^{*}}=0
$$

are of the form

$$
\begin{aligned}
& I_{m}(\alpha \rho) \\
& K_{m}(\alpha \rho)
\end{aligned} e^{-i m \phi} e^{-i h z}
$$

where $\alpha=\left(\gamma^{2}+h^{2}\right)^{\frac{1}{2}}$ and $I_{m}$ and $K_{m}$ are cylindrical Bessel functions of the modified kind defined by G. N. Watson [7].

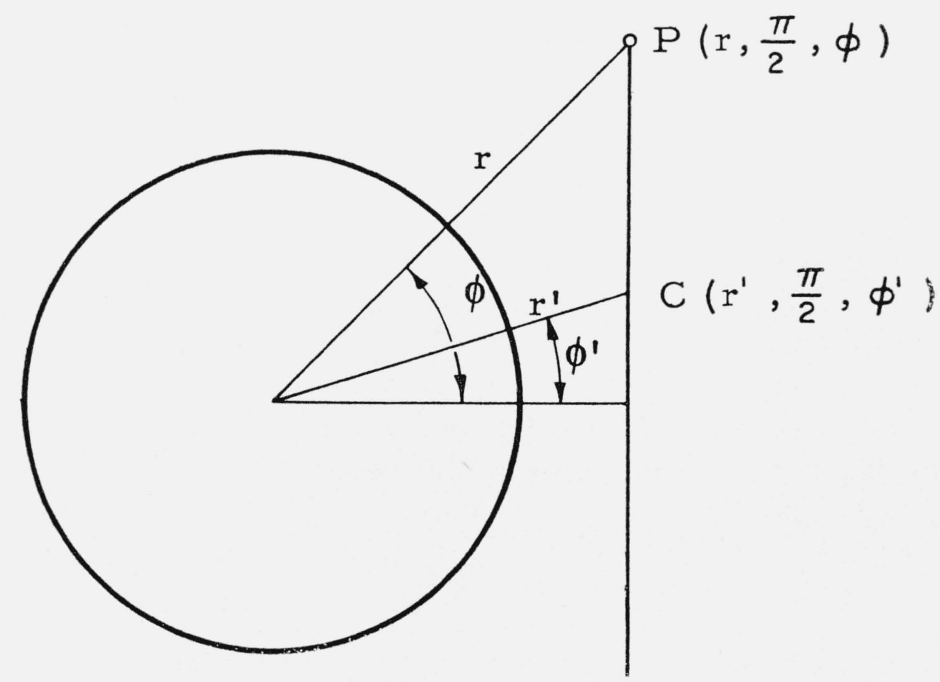

Figure 7.-Special configuration of (dipole) source $C$ and observer $P$ when they are located in equatorial plane of sphere. 


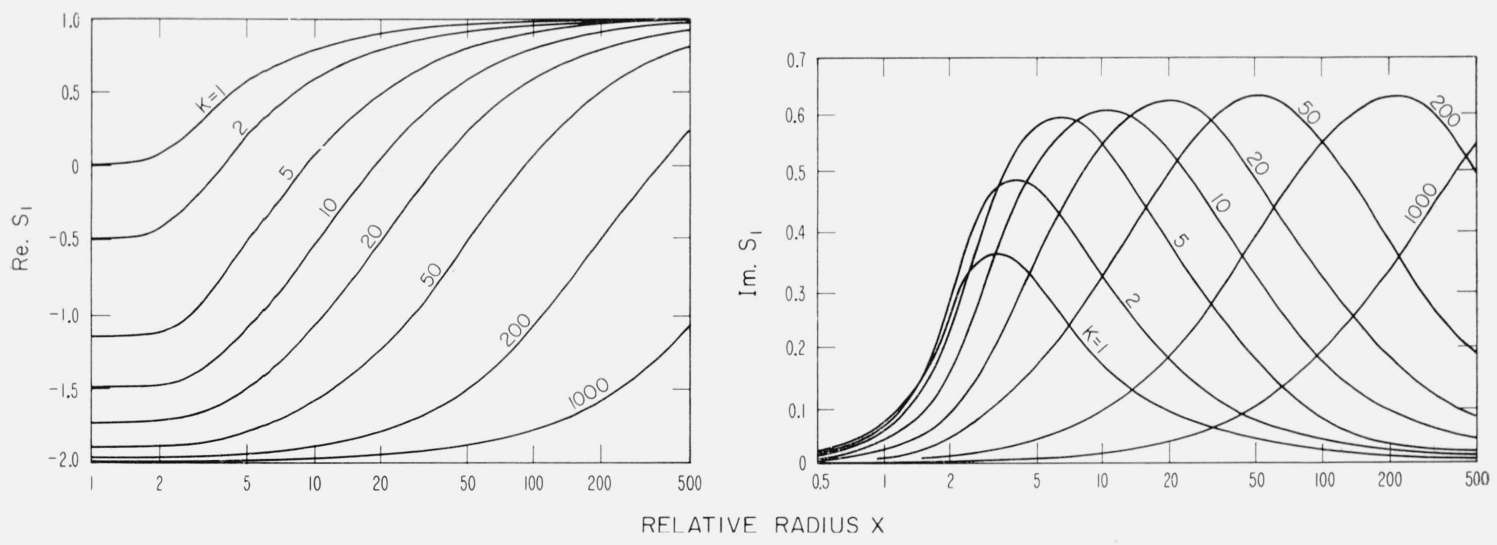

FIGURE 8.- The variation of the response of a conducting sphere as a function of $X\left(=(\sigma \mu \omega)^{\frac{1}{2}} a\right)$ for various permeability ratios $\mathrm{K}\left(=\mu / \mu_{0}\right)$.

The real and imaginary parts of $S_{1}$ are shown.

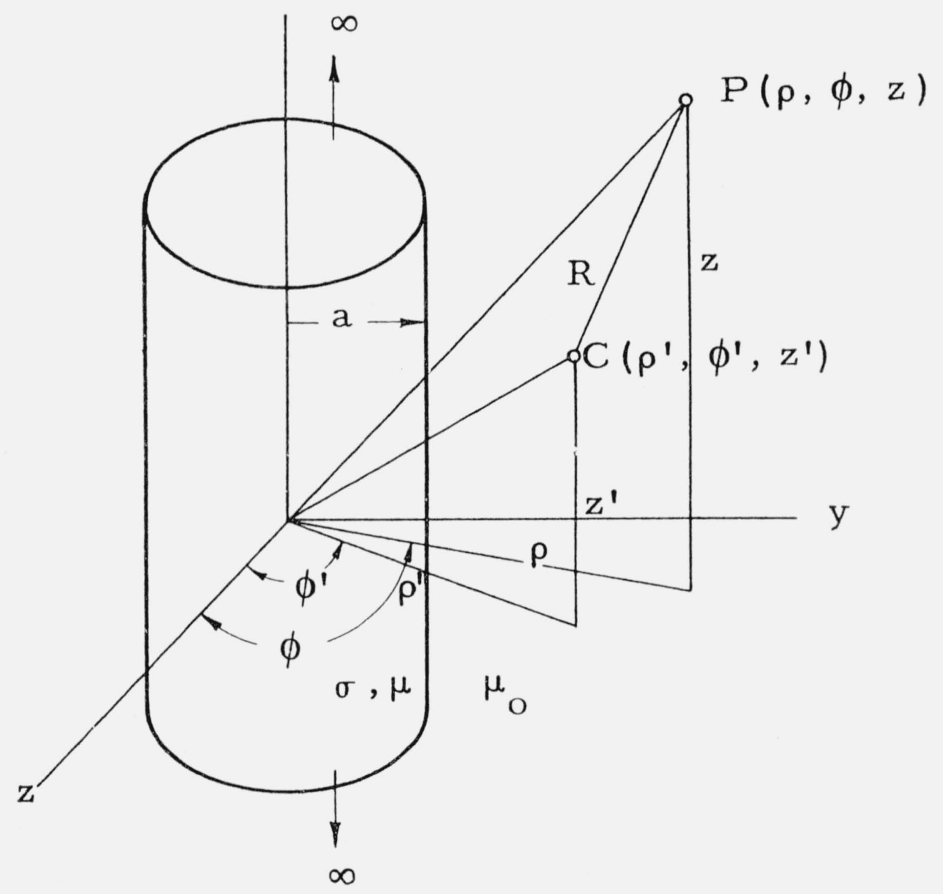

FIGURE 9.-The cylindrical body and source $C$ and observer $P$ with the cylindrical coordinate system.

External to the cylinder $(r>a)$

$$
\vec{H}=-\operatorname{grad} \Omega
$$

where

$$
\Omega=\Omega^{p}+\Omega^{\omega} .
$$

For a magnetic charge at $C\left(\rho^{\prime}, \phi^{\prime}, z^{\prime}\right)$ and the observer at $P(\rho, \phi, z)$,

$$
\Omega^{p}=\frac{\bar{K}}{4 \pi k},
$$




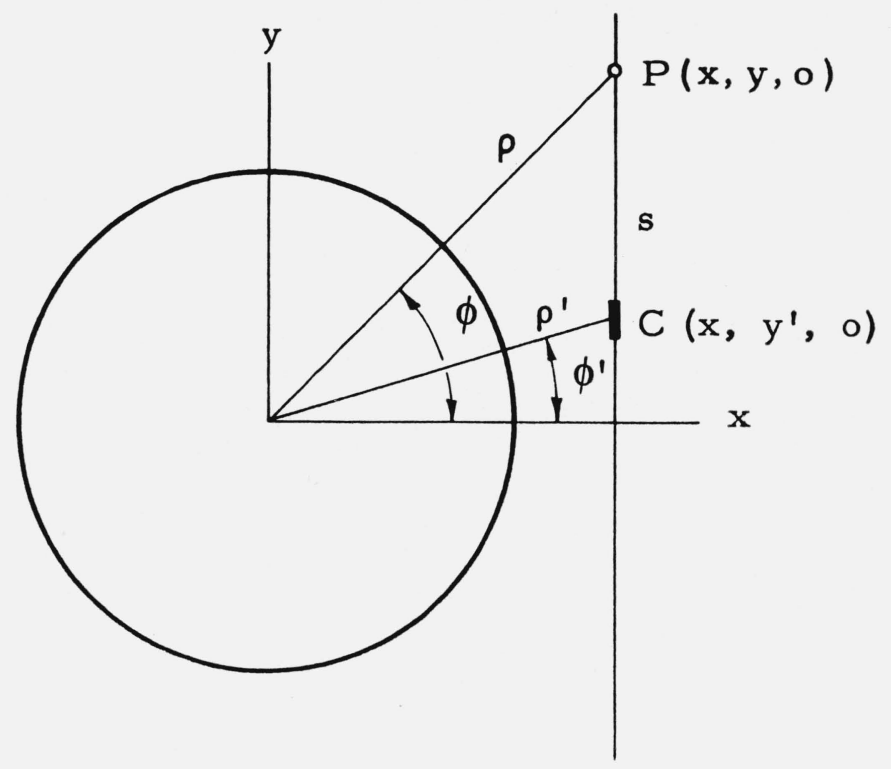

Figure 10.- Special configuration of (dipole) source $C$ and observer $P$ when they are located in equatorial plane of cylinder.

where $R$ is the usual linear distance between $C$ and $P$. Using a formula given by Watson [7] we find

$$
\frac{1}{R}=\frac{1}{\pi} \int_{-\infty}^{+\infty} K_{0}(h \hat{\rho}) e^{-i h\left(z-z^{\prime}\right)} d h
$$

where

$$
R=\sqrt{(\hat{\rho})^{2}+\left(z-z^{\prime}\right)^{2}}
$$

with

$$
\hat{\rho}=\left[\rho^{2}+\left(\rho^{\prime}\right)^{2}-2 \rho \rho^{\prime} \cos \left(\phi-\phi^{\prime}\right)\right]^{\frac{1}{2}} .
$$

Using the addition theorem

for $\rho<\rho^{\prime}$ and with

$$
K_{0}(h \hat{\rho})=\sum_{m=0}^{\infty} \epsilon_{m} K_{m}\left(h \rho^{\prime}\right) I_{m}(h \rho) \cos m\left(\phi-\phi^{\prime}\right)
$$

$$
\epsilon_{0}=1, \quad \epsilon_{m}=2(m=1,2,3 \ldots),
$$

it now readily follows that

$$
\Omega^{p}=\frac{\bar{K}}{4 \pi} \sum_{m=-\infty}^{+\infty} \int_{-\infty}^{+\infty} K_{m}\left(h \rho^{\prime}\right)\left\{I_{m}(h \rho) e^{-i h(z-z \prime)} d h\right\} e^{-i m\left(\phi-\phi^{\prime}\right)} .
$$

This latter equation can be written symbolically in the manner

where $\Gamma$ is the operator

$$
\Omega^{p}=\Gamma I_{m}(h \rho)
$$

$$
\frac{\bar{K}}{4 \pi^{2}} \sum_{m=-\infty}^{+\infty} \int_{-\infty}^{+\infty} K_{m}\left(h \rho^{\prime}\right)\{\ldots\} e^{-i h\left(z-z^{\prime}\right)} d h e^{-i m\left(\phi-\phi^{\prime}\right)} .
$$

This suggests writing

or

$$
\Omega^{s}=\Gamma A_{m}(h) K_{m}(h \rho)
$$

$$
\Omega=\Gamma\left[I_{m}(h \rho)+A_{m}(h) K_{m}(h \rho)\right]
$$


for the external fields and

$$
\begin{aligned}
& \Pi_{z}^{*}=\Gamma a_{m}(h) I_{m}(\alpha \rho), \\
& \Pi_{z}=\Gamma b_{m}(h) I_{m}(\alpha \rho)
\end{aligned}
$$

for the internal fields. The corresponding magnetic field components are

and

$$
\left.\begin{array}{l}
H_{\rho}=-\Gamma h\left[I_{m}^{\prime}(h \rho)+A_{m}(h) K_{m}^{\prime}(h \rho)\right] \\
H_{\phi}=\Gamma \frac{i m}{\rho}\left[I_{m}(h \rho)+A_{m}(h) K_{m}(h \rho)\right] \\
H_{z}=\Gamma i h\left[I_{m}(h \rho)+A_{m}(h) K_{m}(h \rho)\right]
\end{array}\right\} r \geqq a
$$

$$
\begin{aligned}
& H_{p}=\Gamma\left\{-i \hbar \alpha a_{m}(h) I_{m}^{\prime}(\alpha \rho)-\frac{\gamma^{2} m}{\mu \omega \rho} b_{m}(h) I_{m}(\alpha \rho)\right\} \\
& \left.H_{\phi}=\Gamma\left\{-\frac{h m}{\rho} a_{m}(h) I_{m}(\alpha \rho)-\frac{\gamma^{2} a}{i \mu \omega} b_{m}(h) I_{m}^{\prime}(\alpha \rho)\right\}\right\} r \leqq a \\
& H_{z}=\Gamma\left\{-\alpha^{2} a_{m}(h) I_{m}(\alpha \rho)\right\}
\end{aligned}
$$

The unknown coefficients $a_{m}, b_{m}$, and $A_{m}$, which are functions of $h$, are determined from the boundary conditions. These are the normal flux, (i.e., $\mu H_{\rho}$ ) and the tangential fields, (i.e., $H_{\phi}$ and $H_{z}$ ) are continuous at $r=a$. The explicit result for $A_{m}(h)$ is found to be

where

$$
A_{m}(h)=-\left[\frac{\tilde{I}_{m}(h a)-\left(\mu / \mu_{0}\right)\left[\tilde{I}_{m}(\alpha a)+\frac{m^{2} \gamma^{2}}{\alpha^{4} h^{2} a^{4}} \frac{1}{\tilde{I}_{m}(\alpha a)}\right]}{\tilde{K}_{m}(h a)-\left(\mu / \mu_{0}\right)\left[\tilde{I}_{m}(\alpha a)+\frac{m^{2} \gamma^{2}}{\alpha^{4} h^{2} a^{4}} \frac{1}{\tilde{I}_{m}(\alpha a)}\right]}\right] \frac{I_{m}(h a)}{K_{m}(h a},
$$

and

$$
\tilde{I}_{m}(\alpha)=\frac{I_{m}^{\prime}(\alpha)}{\alpha I_{m}(\alpha)}
$$

$$
\tilde{K}_{m}(\alpha)=\frac{K_{m}^{\prime}(\alpha)}{\alpha K_{m}(\alpha)}
$$

The preceding results can now be employed to calculate the magnetic field of a magnetic dipole. In the case of a $y$-directed dipole at $C$ the potential at $P$ is $-d l \partial \Omega / \partial y^{\prime}$. The $y$-component of the field at $P$ is obtained from

$$
H_{y}=+d l \frac{\partial^{2} \Omega}{\partial y \partial y^{\prime}} .
$$

Restricting our attention again to the plane $z=z^{\prime}=0$ and for $x=x^{\prime}$ (see fig. 10), we have

$$
H_{y}^{p}=-\frac{\overline{1} \boldsymbol{d} l l}{2 \pi s^{3}},
$$

where $s=y-y^{\prime}>0$. Noting that

$$
\frac{\partial}{\partial y}=\sin \phi \frac{\partial}{\partial \rho}+\cos \phi \frac{\partial}{\rho \partial \phi}
$$

it readily follows that

$$
\begin{aligned}
H_{y}^{s}=\frac{\bar{K} d l}{4 \pi^{2}} \sum_{m=-\infty}^{+\infty} \int_{-\infty}^{+\infty} A_{m}(h) & {\left[h K_{m}^{\prime}(h \rho) \sin \phi-\frac{i m}{\rho} K_{m}(h \rho) \cos \phi\right] } \\
& \times\left[h K_{m}^{\prime}(h \rho) \sin \phi^{\prime}+\frac{i m}{\rho^{\prime}} K_{m}\left(h \rho^{\prime}\right) \cos \phi^{\prime}\right] e^{-i h\left(z-z^{\prime}\right)} d h e^{-i m\left(\phi-\phi^{\prime}\right)},
\end{aligned}
$$

where $A_{m}(h)$ is given explicitly above. 


\section{A Related Cylindrical Problem}

The above general solution for an infinitely long cylinder with dipolar excitation is rather complicated. The presence of the infinite integrals and the existence of Bessel functions of generally complex argument complicate the numerical aspects of the problem. It appears, however, that simplifications can be made under certain limiting conditions. For example, if the radius $a$ is small and at low frequencies, $h a$ is much less than unity over the important range of the integration. Thus,

$$
A_{m}(h) \cong-\left[\frac{m K I_{m}(\gamma a)-(\gamma a) I_{m}^{\prime}(\gamma a)}{m K I_{m}(\gamma a)+(\gamma a) I_{m}^{\prime}(\gamma a)}\right] \frac{I_{m}(h a)}{K_{m}(h a)},
$$

where $K=\mu / \mu_{0}$. In the case of very high conductivity $|\gamma a|>>1$ this simplifies further to

$$
A_{m}(h) \cong-\frac{I_{m}(h a)}{K_{m}(h a)} .
$$

The square bracket term in equation (81) thus characterizes the frequency dependence of the response and is a function only of $K$ and $\gamma a$.

The secondary magnetic field $H^{s}$ for the case of finite $\gamma a$ can then be written in terms of value of $\vec{H}^{s}$ for infinite $\gamma a$ in the following way (for a given mode number $m$ )

$$
\frac{\vec{H}^{s}}{\left[\vec{H}^{s}\right] \gamma a \rightarrow \infty} \simeq T_{m} \quad(m=1,2,3, \ldots),
$$

where

$$
T_{m}=-\left[\frac{m K I_{m}(\sqrt{i} X)-\sqrt{i} X I_{m}^{\prime}(\sqrt{i} X)}{m K I_{m}(\sqrt{i} X)+\sqrt{i} X I_{m}^{\prime}(\sqrt{i} X)}\right]
$$

and where $X=(\sigma \mu \omega)^{\frac{1}{2}} a$.

The total field is, of course, a sum over all modes, but because of the factor $I_{m}(h a) / K_{m}(h a)$, the relative response decreases rapidly with increasing value of $m$. This follows from the approximation

$$
\frac{I_{m}(h a)}{K_{m}(h a)} \cong \frac{(h a)^{2 m}}{(m !)(m-1) ! 2^{2 n-1}},
$$

which is valid for $(h a)^{2} \ll 1$.

To shed further light on the above approximate form, it is constructive to examine a related problem. If the source is an infinite line source of electric current $I_{0}$ located at $\left(\rho_{0}, \phi_{0}\right)$ and parallel to the z-axis, it is known that the secondary fields due to the presence of the cylinder are [10]

$$
H_{\rho}^{s}=-\frac{I_{0}}{2 \pi} \sum_{m=1}^{\infty} T_{m} \frac{a^{2 m}}{\rho_{0}^{m} \rho^{m+1}} \sin m\left(\phi-\phi_{0}\right)
$$

and

$$
H_{\phi}^{s}=-\frac{I_{0}}{2 \pi} \sum_{m=1}^{\infty} T_{m} \frac{a^{2 m}}{\rho_{0}^{m} \rho^{m+1}} \cos m\left(\phi-\phi_{0}\right)
$$


The structure of this exact two-dimensional solution is very similar to the approximated form of the three-dimensional solution mentioned above. Apparently if $a$ is reasonably small, the induced currents flow mainly in the axial direction even for a localized excitation and thus the frequency dependence of the induced eddy currents are adequately described by the form of the solution of the corresponding two-dimensional problem.

The numerical values of the real and imaginary parts of the function $T_{m}$ are illustrated in figure 11, where they are plotted as a function of $X$, the "relative radius" of the cylinder, for various values of the permeability ratio $K$. It is noted that the factor $T_{1}$, corresponding to the dominant mode, is very similar to the factor $S_{1}$ for the sphere shown in figure 8 .
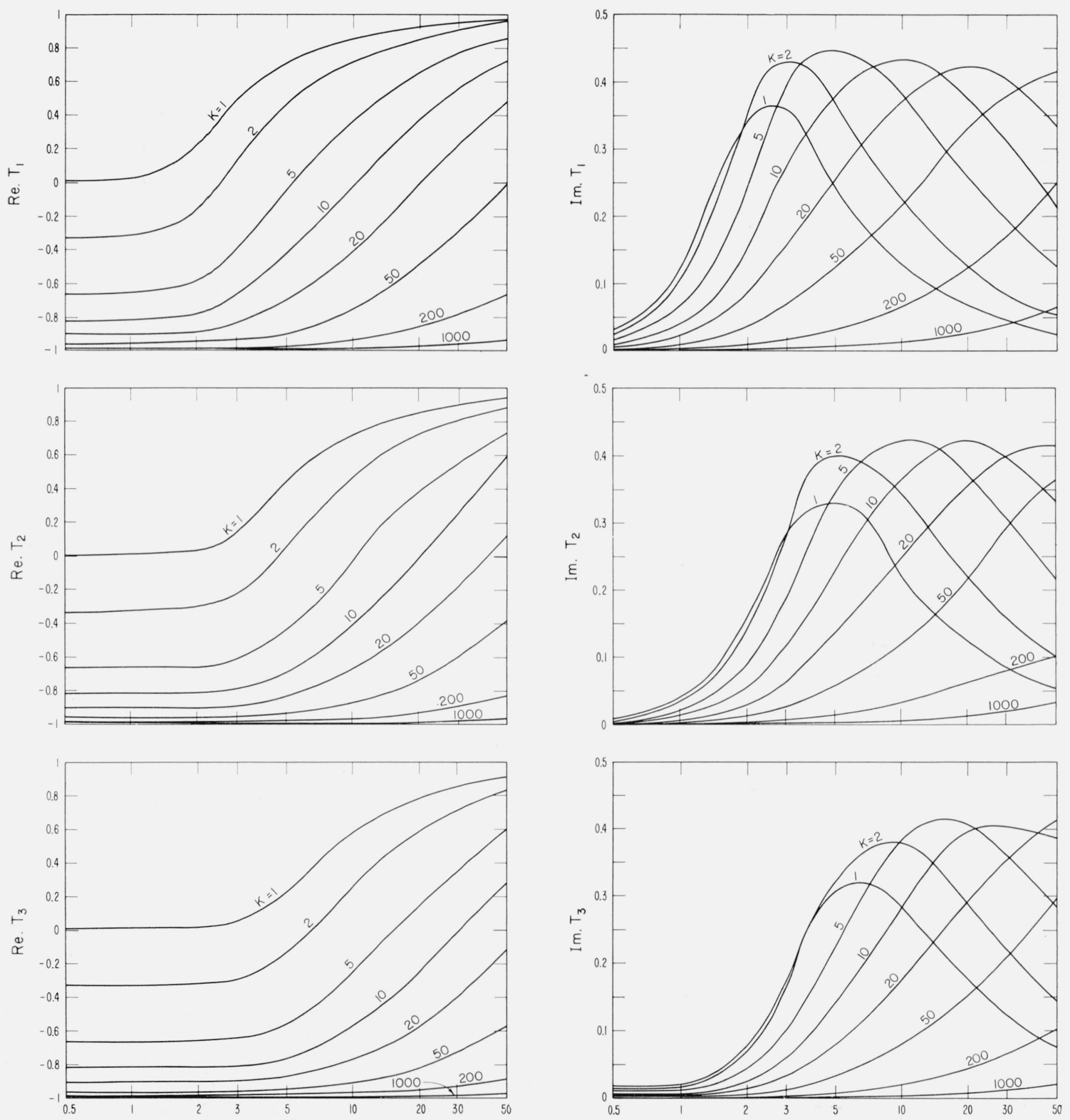

RELATIVE RADIUS $x$

FIGURE 11.-The variation of the response of a conducting cylinder as a function of $X\left(=(\sigma \mu \omega)^{\frac{1}{2}}\right.$ a) for various permeability ratios $K\left(=\mu / \mu_{0}\right)$.

The real and imaginary parts of $T_{1}, T_{2}$, and $T_{3}$ are shown. 
The work in this paper was carried out while the author was employed by Newmont Exploration Limited, Danbury, Conn. The author thanks Drs. A. A. Brant and N. F. Ness of this organization for their helpful suggestions and advice.

\section{References}

[1] C. A. Heiland, Geophysical exploration (Prentice-Hall, Inc., New York, N.Y., 1940).

[2] J. R. Wait, A conducting sphere in a time varying magnetic field, Geophysics 16, 666 (1951).

[3] J. R. Wait, Mutual electromagnetic coupling of loops over a homogeneous ground, Geophysics 20, 630 (1955); contains many references to related work.

[4] S. H. Ward and H. A. Harvey, Electromagnetic surveying of diamond drill holes, Can. Mining Manual, 1 (1954).

[5] W. R. Smythe, Static and dynamic electricity, 2d ed. (McGraw-Hill Book Co., New York, N.Y., 1950); such functions are tabulated in Tables of associated Legendre polynomials (Columbia Univ. Press, New York, N.Y.).

[6] S. A. Schelkunoff, Electromagnetic waves (D. Van Nostrand Co., Inc., New York, N.Y., 1943).

[7] G. N. Watson, Theory of Bessel functions (Cambridge Univ. Press, England, 1945).

[8] S. H. Ward, A method for measuring the electrical conductivity of diamond drill core specimens, Geophysics 18, $434(1953)$.

[9] J. R. Wait, A conducting permeable sphere in the presence of a coil carrying an oscillating current, Can. J. Phys. 31, 670 (1953).

[10] J. R. Wait, The cylindrical ore body in the presence of a cable carrying an oscillating current, Geophysics 17, 378 (1952).

Boulder, Colo.

(Paper 64B1-16) 